\title{
A Literature Review of Ambivalence in Consumer Research: An Abstract
}

\author{
Jenni Sipilä, Sanna Sundqvist, and Anssi Tarkiainen
}

\begin{abstract}
Despite the importance of ambivalence in consumer behavior, its varying and fragmented definitions have made it challenging to understand what ambivalence really means to consumer researchers. This is problematic because the lack of precise conceptualization hinders scientific progress (Teas and Palan 1997) and leads to validity and measurement issues, ill-advised understanding of relationships between concepts, and reduced credibility of research findings (MacKenzie 2003). Thus, in search for greater clarity of the consumer ambivalence concept, this paper first analyzes how ambivalence has been defined in consumer research and subsequently divides the definitions into categories and subcategories of consumer ambivalence. Systematic literature review is used to meet this goal, following a standard procedure of (1) database search with the keyword combinations "consumer" and "ambivalence" and "consumer" and "mixed emotions," (2) exclusion of papers not in scope of the review based on predefined exclusion criteria, and (3) analysis of the conceptualizations used in the papers. Based on this process, a categorization of different types of ambivalences is developed, including the categories of attitudinal ambivalence, mixed emotions, and consumer culture theory-based ambivalence and their subcategories. Finally, a synthesizing view of consumer ambivalence is suggested based on the review and categorization of the definitions, which takes into account the wide spectrum of consumer research. This paper thus advances theoretical discussion of ambivalence in consumer research by providing a clear structure for what is meant by ambivalence in different streams of consumer research, and how these conceptualizations can be synthesized under the umbrella of consumer ambivalence. Finally, a research agenda for consumer ambivalence is developed, including suggestions for more thorough research on the temporal aspects of consumer ambivalence as they relate to consumer decision-making processes, the measurement of ambivalence, and the relationships between consumer ambivalence and other types of consumption-related conflicts.
\end{abstract}

References Available Upon Request

J. Sipilä $(\triangle) \bullet$ S. Sundqvist • A. Tarkiainen

School of Business and Management, Lappeenranta University of Technology,

Lappeenranta, Finland

e-mail: jenni.sipila@lut.fi; sanna.sundqvist@lut.fi; anssi.tarkiainen@lut.fi

(C) Academy of Marketing Science 2017

P. Rossi (ed.), Marketing at the Confluence between Entertainment and Analytics, Developments in Marketing Science: Proceedings of the Academy of Marketing Science, DOI 10.1007/978-3-319-47331-4_173 